\title{
Analytic continuation of multiple polylogarithms *
}

\author{
Jianqiang Zhao ${ }^{\dagger}$
}

\begin{abstract}
In this paper we shall define the analytic continuation of the multiple polylogarithms by using Chen's theory of iterated path integrals and compute the monodromy of all multiple logarithms explicitly.
\end{abstract}

\section{Introduction}

In recent years, there is a revival of interest in multi-valued classical polylogarithms and their single-valued cousins. For any positive integer $m_{1}, \ldots, m_{n}$, Goncharov [6] generalizes the classical polylogarithms and defines the multiple polylogarithms as follows:

$$
L i_{m_{1}, \ldots, m_{n}}\left(x_{1}, \ldots, x_{n}\right)=\sum_{0<k_{1}<k_{2}<\cdots<k_{n}} \frac{x_{1}^{k_{1}} x_{2}^{k_{2}} \ldots x_{n}^{k_{n}}}{k_{1}^{m_{1}} k_{2}^{m_{2}} \ldots k_{n}^{m_{n}}}, \quad\left|x_{i}\right|<1 .
$$

Conventionally one refers $n$ as the depth and $K:=m_{1}+\cdots+m_{n}$ as the weight. When the depth $n=1$ the function is nothing but the classical polylogarithm. More than a century ago it was already known to H. Poincaré [11] that hyperlogarithms

$$
F_{n}\left(\begin{array}{c}
a_{1}, \ldots, a_{n} \\
b_{1}, \ldots, b_{n}
\end{array} \mid z\right)=\int_{b_{n}}^{z} \cdots \int_{b_{2}}^{t_{3}} \int_{b_{1}}^{t_{2}} \frac{d t_{1}}{t_{1}-a_{1}} \frac{d t_{2}}{t_{2}-a_{2}} \cdots \frac{d t_{n}}{t_{n}-a_{n}}
$$

are important for solving differential equations. Notice that the multiple polylogarithm

$$
L i_{m_{1}, \ldots, m_{n}}\left(x_{1}, \ldots, x_{n}\right)=(-1)^{n} F_{K}\left(\begin{array}{ccc}
a_{1}, \overbrace{0, \ldots, 0}^{m_{1}-1 \text { times }}, \ldots, a_{n}, \overbrace{0, \ldots, 0}^{m_{n}-1 \text { times }} \\
0,0, \ldots, 0, \ldots, 0,0, \ldots, 0
\end{array} \mid 1\right)
$$

where $a_{i}=1 /\left(x_{i} \ldots x_{n}\right)$ for $1 \leq i \leq n$. It is an iterated path integral in the sense of Chen [3] whose path lies in $\mathbb{C}$. One thus can easily enlarge its domain of definition to some open subset of $\mathbb{C}^{n}$. However, it is not obvious that this actually gives a genuine analytic continuation in the usual sense. In particular, it is difficult to study the monodromy of the multiple polylogarithms by this expression. It is our primary goal in this paper to define the analytic continuation of the above function to $\mathbb{C}^{n}$ as a multi-valued meromorphic function by using Chen's iterated path integrals with all paths lying in $\mathbb{C}^{n}$.

In early 1980s Deligne [5] discovers that the dilogarithm gives rise to a good variation of mixed Hodge-Tate structures. This has been generalized to polylogarithms (cf. [9]) following Ramakrishnan's computation of the monodromy of the polylogarithms. The monodromy computation also yields the single-valued variant $\mathcal{L}_{n}(z)$ of the polylogarithms (cf. [1, 15]). These functions in turn have significant applications in arithmetic such as Zagier's conjecture $[15$, p.622]. On the other hand, as pointed out in $[7,8]$, "higher cyclotomy theory" should study the multiple polylogarithm motives at roots of unity, not only those of the polylogarithms. This is the primary reason for us to focus our attention on multiple polylogarithms.

\footnotetext{
*Mathematics Subject Classification (2000): 32D15, 53C65, 14D05, 33B30.

${ }^{\dagger}$ Partially supported by NSF grant DMS0139813
} 
According to the theory of framed mixed Hodge-Tate structures the multiple polylogarithms are period functions of some variations of mixed Hodge-Tate structures (see [2], [6, $\S 12]$ and $[6, \S 3.5])$. However, in order to have "reasonable" variations we should be able to control their behavior at "infinity" $X_{n}$ (see (3)). Our analytic continuation of the multiple polylogarithms is more straight-forward than (2) so that we can not only see that the multiple polylogarithms are multi-valued but also determine $X_{n}$ explicitly where the singularities lie. We then compute the monodromy of all multiple logarithms

$$
\mathfrak{L}_{n}\left(x_{1}, \ldots, x_{n}\right):=L i \underbrace{1, \ldots, 1}_{n \text { times }}\left(x_{1}, \ldots, x_{n}\right) .
$$

This enables us to calculate mixed Hodge structures associated with some families of multiple polylogarithms in another paper [16] including all the multiple logarithms.

We point out that the mixed Hodge structures of iterated integrals over $\mathbb{C P} \backslash\{0,1, \infty\}$ have been studied by Wojtkowiak [14]. His approach is completely different from ours.

The motivation of this paper comes from $[7, \S 2,3]$ where the Hodge-Tate structures associated with the double logarithms are discussed. The author thanks his advisor Sasha Goncharov for his constant encouragement and Herbert Gangl for kindly informing the author of the preprint [14] of Wojtkowiak.

\section{Preliminaries on Chen's theory of iterated path inte- grals}

The main references of this section are two of Chen's papers [3] and [4].

For a 1 -form $f(t) d t$ over $\mathbb{R}$ the integral $\int_{a}^{b} f(t) d t$ is understood in the usual way. For $r>1$, define inductively

$$
\int_{a}^{b} f_{1}(t) d t \cdots f_{r}(t) d t=\int_{a}^{b}\left(\int_{a}^{t} f_{1}(\tau) d \tau \cdots f_{r-1}(\tau) d \tau\right) f_{r}(t) d t .
$$

When $r=0$, set the integral to be 1 . For example, the classical polylogarithm

$$
L i_{n}(x)=\int_{0}^{x} \frac{d t}{1-t} \underbrace{\frac{d t}{t} \cdots \frac{d t}{t}}_{(n-1) \text { times }} .
$$

More generally, let $w_{1}, w_{2}, \ldots$ be 1 -forms on a manifold $M$ and let $\alpha:[0,1] \rightarrow M$ be a piecewise smooth path. Write

$$
\alpha^{*} w_{i}=f_{i}(t) d t
$$

and define the iterated path integral

$$
\int_{\alpha} w_{1} \cdots w_{r}=\int_{0}^{1} f_{1}(t) d t \cdots f_{r}(t) d t
$$

The following results are crucial for the application of the Chen's theory of iterated path integrals.

Lemma 2.1. Let $w_{i}(i \geq 1)$ be $\mathbb{C}$-valued 1-forms on a manifold $M$.

(i) The value of $\int_{\alpha} w_{1} \cdots w_{r}$ is independent of the parameterization of $\alpha$.

(ii) If $\alpha, \beta:[0,1] \longrightarrow M$ are composable paths (i.e. $\alpha(1)=\beta(0)$ ), then

$$
\int_{\alpha \beta} w_{1} \cdots w_{r}=\sum_{j=0}^{r} \int_{\alpha} w_{1} \cdots w_{i} \int_{\beta} w_{i+1} \cdots w_{r} .
$$


Here, we set $\int_{\alpha} \phi_{1} \cdots \phi_{m}=1$ if $m=0$.

(ii) For every path $\alpha$,

$$
\int_{\alpha^{-1}} w_{1} \cdots w_{r}=(-1)^{r} \int_{\alpha} w_{r} \cdots w_{1}
$$

(iv) For every path $\alpha$,

$$
\int_{\alpha} w_{1} \cdots w_{r} \int_{\alpha} w_{r+1} \cdots w_{r+s}=\sum_{\sigma} \int_{\alpha} w_{\sigma(1)} \cdots w_{\sigma(r+s)}
$$

where $\sigma$ ranges over all shuffles of type $(r, s)$, i.e., permutations $\sigma$ of $r+s$ letters with $\sigma^{-1}(1)<\cdots<\sigma^{-1}(r)$ and $\sigma^{-1}(r+1)<\cdots<\sigma^{-1}(r+s)$.

Proof. (i) can be derived from the theorem on [3, p. 361]. (ii) and (iii) are formulas (1.6.1) and (1.6.2) of [3] respectively. Ree [13] discovered the shuffle relation (iv) which appeared as (1.5.1) in [3].

Lemma 2.2. If $w_{i}^{(j)}$ are closed 1-forms for $1 \leq i \leq r$ and $1 \leq j \leq n$ such that $\sum_{j} w_{1}^{(j)} \wedge$ $w_{2}^{(j)}=\sum_{j} w_{2}^{(j)} \wedge w_{3}^{(j)}=\cdots=\sum_{j} w_{r-1}^{(j)} \wedge w_{r}^{(j)}=0$ then $\sum_{j} \int_{\alpha} w_{1}^{(j)} w_{2}^{(j)} \cdots w_{r}^{(j)}$ only depends on the homotopy class of $\alpha$.

Proof. The case $j=1$ is proved on [3, p. 366]. The case $r=2$ can be found on [3, p. 368]. The general case follows from a similar argument.

\section{The index set $\mathfrak{S}\left(m_{1}, \ldots, m_{n}\right)$}

We first introduce an index set with two different kinds of orderings.

2.1. Definition. Define the index set

$$
\mathfrak{S}\left(m_{1}, \ldots, m_{n}\right)=\left\{\mathbf{i}=\left(i_{1}, \ldots, i_{n}\right): 0 \leq i_{t} \leq m_{t} \text { for } t=1, \cdots, n\right\}
$$

and the weight function $|\cdot|$ on $\mathfrak{S}\left(m_{1}, \ldots, m_{n}\right)$ by

$$
\left|\left(i_{1}, \ldots, i_{n}\right)\right|=i_{1}+\cdots+i_{n} .
$$

For brevity, we write $\mathbf{0}=(0, \ldots, 0) \in \mathfrak{S}\left(m_{1}, \ldots, m_{n}\right)$ which is the only index of weight 0 in $\mathfrak{S}\left(m_{1}, \ldots, m_{n}\right)$ and $\mathbf{1}_{K}=\left(m_{1}, \ldots, m_{n}\right) \in \mathfrak{S}\left(m_{1}, \ldots, m_{n}\right)$ which is the only index of the highest weight $K:=m_{1}+\cdots+m_{n}$ in $\mathfrak{S}\left(m_{1}, \ldots, m_{n}\right)$. We also define the depth function of the index $\left(i_{1}, \ldots, i_{n}\right)$ by $\sharp\left\{t: i_{t} \neq 0\right\}$, i.e., the number of nonzero components.

We shall need two orderings on $\mathfrak{S}\left(m_{1}, \ldots, m_{n}\right)$.

2.2. A complete ordering. The complete ordering is defined as follows. Let $\mathbf{i}=\left(i_{1}, \ldots, i_{n}\right)$ and $\mathbf{j}=\left(j_{1}, \ldots, j_{n}\right)$. If $|\mathbf{i}|<|\mathbf{j}|$ then $\mathbf{i}<\mathbf{j}$ (or, equivalently, $\left.\mathbf{j}>\mathbf{i}\right)$. If $|\mathbf{i}|=|\mathbf{j}|$ then $\mathbf{i}>\mathbf{j}$ if $\max \left\{i_{t}: 1 \leq t \leq n\right\}>\max \left\{j_{t}: 1 \leq t \leq n\right\}$. Otherwise, we compare the second largest components of $\mathbf{i}$ and $\mathbf{j}$, and so on. If $\left\{i_{1}, \ldots, 1_{n}\right\}=\left\{j_{1}, \ldots, j_{n}\right\}$ as two set then the usual lexicographic order from left to right is in force with $0<1<\cdots$. For instance, $(0,0,1)<(1,0,1)<(1,1,0)<(0,2,0)$ in $\mathfrak{S}(1,2,1)$.

Remark 3.1. In the multiple logarithm case, namely, when $m_{1}=\cdots=m_{n}=1$, there is a one-to-one correspondence between $\mathfrak{S}_{n}$ and the set of non-negative integers less than $2^{n}$. Thus one is tempted to use the conventional order of positive integers in binary forms. However this is not suitable in our situation. 
2.3. A partial ordering and the retraction map. The partial ordering is defined as follows. Let $\mathbf{i}=\left(i_{1}, \ldots, i_{n}\right)$ and $\mathbf{j}=\left(j_{1}, \ldots, j_{n}\right)$. We set $\mathbf{j} \prec \mathbf{i}$ (or, equivalently, $\mathbf{i} \succ \mathbf{j}$ ) if $j_{t} \leq i_{t}$ for every $1 \leq t \leq n$. For example $(0,0,1,0) \prec(0,1,1,0)$ in $\mathfrak{S}(1,1,1,1)$ but $(1,0,0,0) \nprec(0,1,1,0)$ and $(1,0,0,0) \nsucc(0,1,1,0)$. Clearly $\mathbf{j} \prec \mathbf{i}$ implies $\mathbf{j}<\mathbf{i}$ but not vice versa.

Suppose $\mathbf{i}$ has depth $k$ with $i_{\tau_{s}} \neq 0$ for $1 \leq s \leq k$ while $\mathbf{j}$ has depth $l$ and $j_{t_{r}} \neq 0$ for $1 \leq r \leq l$. If $\mathbf{j} \prec \mathbf{i}$ then we can write $t_{r}=\tau_{\alpha_{r}}$ for $1 \leq r \leq l$. For such $\mathbf{i}$ and $\mathbf{j}$ we define the $\mathbf{i}$-th retraction map $\rho_{\mathbf{i}}$ from $\mathfrak{S}\left(m_{1}, \ldots, m_{n}\right)$ to $\mathfrak{S}\left(i_{\tau_{1}}, \ldots, i_{\tau_{k}}\right)$ as follows. The entry of $\rho_{\mathbf{i}}(\mathbf{j})$ is $j_{\tau_{\alpha_{r}}}$ if it is at the $\alpha_{r}$-th $(1 \leq r \leq l)$ component and 0 at all other components. For instance $\rho_{(02010)}((01000))=(10) \in \mathfrak{S}(2,1)$. In particular, $\rho_{\mathbf{i}}(\mathbf{i})=\left(i_{\tau_{1}}, \ldots, i_{\tau_{k}}\right)$ has highest weight in $\mathfrak{S}\left(i_{\tau_{1}}, \ldots, i_{\tau_{k}}\right)$.

2.4. Vector indices. Let $\mathfrak{S}^{K}\left(m_{1}, \ldots, m_{n}\right)$ be the set of $K$-tuples $\overrightarrow{\mathbf{j}}=\left(\mathbf{j}_{1}, \ldots, \mathbf{j}_{K}\right)$ of $\mathfrak{S}\left(m_{1}, \ldots, m_{n}\right)$ such that $\left|\mathbf{j}_{t}\right|=t$ and $\mathbf{j}_{1} \prec \cdots \prec \mathbf{j}_{K}=\mathbf{1}_{K}$. One may think $\overrightarrow{\mathbf{j}}$ as a length $K$ queue of indices of $\mathfrak{S}\left(m_{1}, \ldots, m_{n}\right)$ in which each index is produced by increasing some component of the preceding index by 1 .

2.5. Additional notation. Throughout the paper we fix $\mathbf{u}_{s}:=(0, \ldots, 0,1,0, \ldots, 0) \in$ $\mathfrak{S}\left(m_{1}, \ldots, m_{n}\right)$ of weight 1 where the entry 1 is at the $s$-th component. Whenever the $s$-th component $i_{s}$ of $\mathbf{i}$ satisfies $i_{s}<m_{s}$ we can increase $i_{s}$ by 1 to get a new index which is denoted by $\mathbf{i}+\mathbf{u}_{s}$. If $i_{s}>0$ we similarly define $\mathbf{i}-\mathbf{u}_{s}$ as the index with the $s$-th component of $\mathbf{i}$ decreased by 1 . Fix $\mathbf{v}_{s}=\mathbf{1}_{K}-m_{s} \mathbf{u}_{s} \in \mathfrak{S}\left(m_{1}, \ldots, m_{n}\right)$ whose components are nonzero except at the $s$-th position.

When $m_{1}=\cdots=m_{n}=1$ we write $\mathfrak{S}(1, \ldots, 1)=\mathfrak{S}_{n}$.

2.6. Transposition functions. Fix arbitrary $\overrightarrow{\mathbf{J}}=\left(\mathbf{j}_{1}, \ldots, \mathbf{j}_{K}\right) \in \mathfrak{S}^{K}\left(m_{1}, \ldots, m_{n}\right)$ and $1<r \leq K$ we write

$$
\mathbf{j}_{r}=\mathbf{j}_{r-1}+\mathbf{u}_{s}=\left(t_{1}, \ldots, t_{s}, 0, \ldots, 0, t_{a}, \ldots, t_{n}\right), \quad 0 \leq s<a \leq n+1, t_{a} \neq 0 .
$$

Here if $a=n+1$ then the last nonzero component of $\mathbf{j}_{r}$ is $t_{s}$. We define the transposition functions on $\mathbf{i}=\left(i_{1}, \ldots, i_{n}\right) \in \mathfrak{S}(m, \ldots, m)$ with $m=\max \left\{m_{1}, \ldots, m_{n}\right\}$ by

$$
T_{0}^{r}=\mathrm{id}, \quad T_{1}^{r}(\mathbf{i})=\left(i_{\sigma(1)}, \ldots, i_{\sigma(n)}\right)
$$

where if $t_{s}>1$ or $a=n+1$ then $\sigma=$ id whereas if $t_{s}=1$ and $a \leq n$ then $\sigma$ is the transposition in the symmetric group of $n$ elements that exchanges $s$ and $a$.

\section{Analytic continuation of multiple polylogarithms}

Let $\mathbf{x}=\left(x_{1}, \ldots, x_{n}\right)$ be a variable over $\mathbb{C}^{n}$. Define

$$
S_{n}=\mathbb{C}^{n} \backslash X_{n}, \quad S_{n}^{\prime}=\mathbb{C}^{n} \backslash X_{n}^{\prime},
$$

where the divisors are defined by

$$
\begin{aligned}
& X_{n}=\left\{\mathbf{x} \in \mathbb{C}^{n}: \prod_{1 \leq i \leq n}\left(1-x_{j}\right) \prod_{1 \leq j<k \leq n}\left(1-x_{j} \ldots x_{k}\right)=0\right\}, \\
& X_{n}^{\prime}=\left\{\mathbf{x} \in \mathbb{C}^{n}: \prod_{1 \leq i \leq n} x_{j}\left(1-x_{j}\right) \prod_{1 \leq j<k \leq n}\left(1-x_{j} \ldots x_{k}\right)=0\right\} .
\end{aligned}
$$

It is clear that $S_{n}^{\prime} \subset S_{n}$. Set

$$
D_{n}=\left\{\left(x_{1}, \ldots, x_{n}\right) \in \mathbb{C}^{n}:\left|x_{j}-\frac{1}{2}\right|<\frac{1}{2}, j=1, \ldots, n\right\} \subset S_{n}^{\prime} .
$$


Denote a varying base point by $\varepsilon_{n}=(\varepsilon, \ldots, \varepsilon) \in D_{n}$.

Suppose the depth of $\mathbf{i}=\left(i_{1}, \ldots, i_{n}\right)$ is $k$ and $i_{\tau_{1}} \neq 0, \ldots, i_{\tau_{k}} \neq 0$. We define

$$
a_{t}=a_{t}(\mathbf{x}):=\left(x_{t} \ldots x_{n}\right)^{-1} \text { for } 1 \leq t \leq n
$$

and

$$
\mathbf{x}(\mathbf{i})=\mathbf{y}=\left(y_{1}, \ldots, y_{k}\right), \quad y_{m}=\prod_{\alpha=\tau_{m}}^{\tau_{m+1}-1} x_{\alpha}=\frac{a_{\tau_{m+1}}(\mathbf{x})}{a_{\tau_{m}}(\mathbf{x})}, \quad 1 \leq m \leq k
$$

with $\tau_{k+1}=n+1$ and $a_{n+1}=1$. We also write $a_{m}(\mathbf{y})=\left(y_{m} \ldots y_{k}\right)^{-1}=a_{\tau_{m}}(\mathbf{x})$. Note that $\mathbf{x}(\mathbf{i}) \in \mathbb{C}^{k}$ which is the reason why we call $k$ the depth of $\mathbf{i}$.

We begin with some 1 -forms which will be used to express the multiple polylogarithms. Take $\overrightarrow{\mathbf{j}} \in \mathfrak{S}^{K}\left(m_{1}, \ldots, m_{n}\right)$ and $\mathbf{j}_{r}=\mathbf{j}_{r-1}+\mathbf{u}_{s}$ as given in $\S 2.6$. For any $\left(\delta_{1}, \ldots, \delta_{K}\right) \in \mathfrak{S}_{K}$, namely, $\delta_{t}=0$ or 1 , let $\mathbf{y}=\mathbf{x}$ if $r=K$ and

$$
\mathbf{y}=\left(y_{1}, \ldots, y_{l}\right)=\mathbf{x}\left(T_{\delta_{r+1}}^{r+1} \circ \cdots \circ T_{\delta_{K}}^{K}\left(\mathbf{j}_{r}\right)\right) \quad \text { if } \quad 1 \leq r<K
$$

where $l$ is the depth of $\mathbf{j}_{r}$ because the transposition functions do not change the depth of an index. We let $t_{\alpha_{1}} \neq 0, \ldots, t_{\alpha_{l}} \neq 0$ and $s=\alpha_{\lambda}$ (because $t_{s} \neq 0$ ) and set

$$
w_{\overrightarrow{\mathbf{J}}}^{r, \delta_{r}}(\mathbf{y}):= \begin{cases}0 & \text { if } t_{s}>1 \text { and } \delta_{r}=1, \\ d y_{\lambda} / y_{\lambda} & \text { if } t_{s}>1 \text { and } \delta_{r}=0, \\ d y_{\lambda} /\left(1-y_{\lambda}\right) & \text { if } t_{s}=1 \text { and } \delta_{r}=0, \\ d y_{\lambda} / y_{\lambda}\left(y_{\lambda}-1\right) & \text { if } \lambda<l, t_{s}=1 \text { and } \delta_{r}=1, \\ 0 & \text { if } \lambda=l, t_{s}=1 \text { and } \delta_{r}=1 .\end{cases}
$$

It is obvious that $w_{\overrightarrow{\mathbf{J}}}^{r, \delta_{r}}(\mathbf{y})$ is always a closed 1-form whose singularities lie only along $X_{n}^{\prime}$.

Proposition 4.1. Let $\int_{p} \bigsqcup_{r=1}^{K} w_{r}$ denote the iterated integral $\int_{p} w_{1} \cdots w_{K}$. Then for every $\mathbf{x} \in D_{n}$

$$
L i_{m_{1}, \ldots, m_{n}}(\mathbf{x})=\lim _{\varepsilon \rightarrow 0} \int_{\boldsymbol{\varepsilon}_{n}} \sum_{\substack{\left(\delta_{1}, \ldots, \delta_{K}\right) \in \mathfrak{S}_{K} \\ \overrightarrow{\mathbf{J}} \in \mathfrak{S}^{K}\left(m_{1}, \ldots, m_{n}\right)}} \bigsqcup_{r=1}^{K} w_{\overrightarrow{\mathbf{J}}}^{r, \delta_{r}}\left(\mathbf{x}\left(T_{\delta_{r+1}}^{r+1} \circ \cdots \circ T_{\delta_{K}}^{K}\left(\mathbf{j}_{r}\right)\right)\right)
$$

where the paths of the iterated integral lie entirely in $D_{n}$

Proof. We prove this by induction on $K$. When $K=1$ this is trivial. Assume $K>1$ and the proposition is true for $K-1$. Using the power series expansion (1) it is straight-forward to check that

$$
d L i_{m_{1}, \ldots, m_{n}}(\mathbf{x})=\sum_{t=1}^{n} d_{t} L i_{m_{1}, \ldots, m_{n}}(\mathbf{x})
$$

where if $m_{t}>1$ then

$$
d_{t} L i_{m_{1}, \ldots, m_{n}}(\mathbf{x})=L i_{m_{1}, \ldots, m_{t-1}, m_{t}-1, m_{t+1}, \ldots, m_{n}}(\mathbf{x}) d x_{t} / x_{t}
$$

whereas if $m_{t}=1$ then

$$
\begin{aligned}
d_{t} L i_{m_{1}, \ldots, m_{n}}(\mathbf{x})=L i_{m_{1}, \ldots, m_{t-1}, m_{t+1}, \ldots, m_{n}}\left(\mathbf{x}\left(\mathbf{v}_{t}\right)\right) d x_{t} /\left(1-x_{t}\right) & \\
& +L i_{m_{1}, \ldots, m_{t-1}, m_{t+1}, \ldots, m_{n}}\left(\mathbf{x}\left(\mathbf{v}_{t+1}\right)\right) d x_{t} / x_{t}\left(x_{t}-1\right) .
\end{aligned}
$$

Here when $t=n$ and $m_{n}=1$ the second term in the sum does not appear. Observe that for any $\overrightarrow{\mathbf{J}} \in \mathfrak{S}^{K}\left(m_{1}, \ldots, m_{t-1}, 1, m_{t+1}, \ldots, m_{n}\right)$ with $\mathbf{j}_{K-1}=\left(m_{1}, \ldots, m_{t-1}, 0, m_{t+1}, \ldots, m_{n}\right)$ and $t<n$ we have $T_{1}^{K}\left(\mathbf{j}_{K-1}\right)=\left(m_{1}, \ldots, m_{t-1}, m_{t+1}, 0, m_{t+2}, \ldots, m_{n}\right)$ and therefore

$$
\mathbf{x}\left(\mathbf{v}_{t+1}\right)=\mathbf{x}\left(m_{1}, \ldots, m_{t}, 0, m_{t+2}, \ldots, m_{n}\right)=\mathbf{x}\left(T_{1}^{K}\left(\mathbf{j}_{K-1}\right)\right) .
$$


Hence

$$
d L i_{m_{1}, \ldots, m_{n}}(\mathbf{x})=\sum_{\delta_{K}=0,1} \sum_{\substack{\left|\mathbf{j}_{K-1}\right|=K-1, \mathbf{j}_{K-1} \in \mathfrak{S}\left(m_{1}, \ldots, m_{n}\right)}} L i_{T_{\delta_{K}}^{K}\left(\mathbf{j}_{K-1}\right)}\left(\mathbf{x}\left(T_{\delta_{K}}^{K}\left(\mathbf{j}_{K-1}\right)\right)\right) w_{\overrightarrow{\mathbf{j}}}^{K, \delta_{K}}(\mathbf{x})
$$

where we write $L i_{\mathbf{v}_{t}}=L i_{m_{1}, \ldots, m_{t-1}, m_{t+1}, \ldots, m_{n}}$.

For $1 \leq t \leq n$ define the embeddings in the obvious way

$$
\iota_{s}: \mathfrak{S}\left(m_{1}, \ldots, m_{s-1}, m_{s}-1, m_{s+1}, \ldots, m_{n}\right) \hookrightarrow \mathfrak{S}\left(m_{1}, \ldots, m_{n}\right)
$$

where when $m_{s}=1$ the left hand side is understood as $\mathfrak{S}\left(m_{1}, \ldots, m_{s-1}, m_{s+1}, \ldots, m_{n}\right)$ which is identified with $\mathfrak{S}\left(m_{1}, \ldots, m_{s-1}, 0, m_{s+1}, \ldots, m_{n}\right)$ as a subset of the right hand side. By abuse of notation, we further define

$$
\begin{aligned}
\iota_{s}: \mathfrak{S}^{K-1}\left(m_{1}, \ldots, m_{s-1}, m_{s}-1, m_{s+1}, \ldots, m_{n}\right) & \longrightarrow \mathfrak{S}^{K}\left(m_{1}, \ldots, m_{n}\right) \\
\left(\mathbf{k}_{1}, \ldots, \mathbf{k}_{K-1}\right) & \longmapsto\left(\mathbf{k}_{1}, \ldots, \mathbf{k}_{K-1}, \mathbf{1}_{K}\right) .
\end{aligned}
$$

By induction

$$
\begin{aligned}
L i_{T_{\delta_{K}}^{K}\left(\mathbf{j}_{K-1}\right)}\left(\mathbf{x}\left(T_{\delta_{K}}^{K}\left(\mathbf{j}_{K-1}\right)\right)\right)=\lim _{\varepsilon \rightarrow 0} & \int_{\boldsymbol{\varepsilon}}^{\mathbf{x}\left(T_{\delta_{K}}^{K}\left(\mathbf{j}_{K-1}\right)\right)} \sum_{\left(\delta_{1}, \ldots, \delta_{K-1}\right) \in \mathfrak{S}_{K-1} \overrightarrow{\mathbf{k}} \in \mathfrak{S}^{K-1}\left(T_{\delta_{K}}^{K}\left(\mathbf{j}_{K-1}\right)\right)} \\
& \bigsqcup_{r=1}^{K-1} w_{\overrightarrow{\mathbf{k}}}^{r, \delta_{r}}\left(\mathbf{x}\left(T_{\delta_{K}}^{K}\left(\mathbf{j}_{K-1}\right)\right)\left(\widetilde{T}_{\delta_{r+1}}^{r+1} \circ \ldots \circ \widetilde{T}_{\delta_{K-1}}^{K-1}\left(\mathbf{k}_{r}\right)\right)\right)
\end{aligned}
$$

where the transposition functions $\widetilde{T}$ may differ from $T$. Let $\mathbf{j}_{K}=\mathbf{j}_{K-1}+\mathbf{u}_{s}$. We now show that

$$
\mathbf{x}\left(T_{\delta_{K}}^{K}\left(\mathbf{j}_{K-1}\right)\right)\left(\widetilde{T}_{\delta_{r+1}}^{r+1} \circ \cdots \circ \widetilde{T}_{\delta_{K-1}}^{K-1}\left(\mathbf{k}_{r}\right)\right)=\mathbf{x}\left(T_{\delta_{r+1}}^{r+1} \circ \cdots \circ T_{\delta_{K}}^{K}\left(\iota_{s}\left(\mathbf{k}_{r}\right)\right)\right) .
$$

This is trivial if $\delta_{K}=0$ or $s=n$ or $t_{s}>1$ where $t_{s}$ is the $s$-th component of $\mathbf{j}_{K}$ because in these cases we have $T_{\delta_{K}}^{K}=\mathrm{id}, \widetilde{T}=T$ and $\iota_{s}=\mathrm{id}$. So we assume $\delta_{K}=1, t_{s}=1$ and $s<n$. Then

$$
\mathbf{x}\left(T_{\delta_{K}}^{K}\left(\mathbf{j}_{K-1}\right)\right)=\left(x_{1}, \ldots, x_{s-1}, x_{s} x_{s+1}, x_{s+2}, \ldots, x_{n}\right)
$$

and the $s$-component of $\iota_{s}\left(\mathbf{k}_{r}\right)$ is 0 by definition. By straight-forward computation we find that

$$
T_{\delta_{K}}^{K} \circ \iota_{s} \circ \widetilde{T}_{\delta_{\lambda}}^{\lambda}=T_{\delta_{\lambda}}^{\lambda} \circ T_{\delta_{K}}^{K} \circ \iota_{s} \quad \text { for all } r+1 \leq \lambda \leq K-1 .
$$

These implies equation (8) immediately because

$$
\begin{aligned}
\mathbf{x}\left(T_{\delta_{K}}^{K}\left(\mathbf{j}_{K-1}\right)\right)\left(\widetilde{T}_{\delta_{r+1}}^{r+1} \circ \cdots \circ \widetilde{T}_{\delta_{K-1}}^{K-1}\left(\mathbf{k}_{r}\right)\right) & =\mathbf{x}\left(T_{\delta_{K}}^{K} \circ \iota_{s} \circ \widetilde{T}_{\delta_{r+1}}^{r+1} \circ \cdots \circ \widetilde{T}_{\delta_{K-1}}^{K-1}\left(\mathbf{k}_{r}\right)\right) \\
& =\mathbf{x}\left(T_{\delta_{r+1}}^{r+1} \circ \cdots \circ T_{\delta_{K}}^{K}\left(\iota_{s}\left(\mathbf{k}_{r}\right)\right)\right) .
\end{aligned}
$$

Therefore from (7) and the one-to-one correspondence:

$$
\iota_{s}: \mathfrak{S}^{K-1}\left(T_{\delta_{K}}^{K}\left(\mathbf{j}_{K-1}\right)\right) \longleftrightarrow\left\{\left(\mathbf{i}_{1}, \ldots, \mathbf{i}_{K}\right) \in \mathfrak{S}^{K}\left(m_{1}, \ldots, m_{n}\right): \mathbf{i}_{K-1}=T_{\delta_{K}}^{K}\left(\mathbf{j}_{K-1}\right)\right\}
$$

we see that $d L i_{m_{1}, \ldots, m_{n}}(\mathbf{x})$ is equal to

$$
\sum_{\substack{\left(\delta_{1}, \ldots, \delta_{K}\right) \in \mathfrak{S}_{K} \\ \overrightarrow{\mathbf{J}} \in \mathfrak{S}^{K}\left(m_{1}, \ldots, m_{n}\right)}}\left(\lim _{\varepsilon \rightarrow 0} \int_{\varepsilon}^{\mathbf{x}\left(T_{\delta_{K}}^{K}\left(\mathbf{j}_{K-1}\right)\right)} \bigsqcup_{r=1}^{K-1} w_{\overrightarrow{\mathbf{J}}}^{r, \delta_{r}}\left(\mathbf{x}\left(T_{\delta_{r+1}}^{r+1} \circ \cdots \circ T_{\delta_{K}}^{K}\left(\mathbf{j}_{r}\right)\right)\right)\right) w_{\overrightarrow{\mathbf{J}}}^{K, \delta_{K}}(\mathbf{x}) .
$$

This finishes the proof of the proposition by induction because $\lim _{\varepsilon \rightarrow 0} L i_{m_{1}, \ldots, m_{n}}\left(\varepsilon_{n}\right)=0$ where the limiting process takes place inside $D_{n}$. 
By the above proposition we can define the analytic continuation of $L i_{m_{1}, \ldots, m_{n}}(\mathbf{x})$ to $S_{n}^{\prime}$ as the iterated path integral

$$
L i_{m_{1}, \ldots, m_{n}}(\mathbf{x})=\lim _{\varepsilon \rightarrow 0} \int_{\boldsymbol{\varepsilon}_{n}} \sum_{\substack{\left(\delta_{1}, \ldots, \delta_{K}\right) \in \mathfrak{S}_{K} \\ \overrightarrow{\mathbf{J}} \in \mathfrak{S}^{K}\left(m_{1}, \ldots, m_{n}\right)}} \bigsqcup_{r=1}^{K} w_{\overrightarrow{\mathbf{J}}}^{r, \delta_{r}}\left(\mathbf{x}\left(T_{\delta_{r+1}}^{r+1} \circ \cdots \circ T_{\delta_{K}}^{K}\left(\mathbf{j}_{r}\right)\right)\right),
$$

where all the paths lie inside $S_{n}^{\prime}$. Note that all the 1-forms appearing in (9) are rational forms with logarithmic singularities along $X_{n}^{\prime}$.

Example 4.2. When $n=1$,

$$
L i_{1}(x)=\int_{0}^{x} d \log \left(\frac{1}{1-x}\right)=-\log (1-x) .
$$

When $n=2, \mathfrak{S}_{2}=\{(0,0),(0,1),(1,0),(1,1)\}$ and there are two elements in $\mathfrak{S}^{2}:((0,1),(1,1))$ and $((1,0),(1,1))$. Let $\mathbf{x}=(x, y)$ then

$$
\mathbf{x}(0,1)=y, \quad \mathbf{x}(1,0)=x y, \quad \mathbf{x}(1,1)=(x, y) .
$$

Thus

$$
\begin{aligned}
L i_{1,1}(x, y) & =\int_{\mathbf{0}}^{\mathbf{x}} w_{1}(\mathbf{x}(0,1)) w_{1}(\mathbf{x})+w_{1}(\mathbf{x}(1,0)) w_{2}(\mathbf{x}) \\
& =\int_{(0,0)}^{(x, y)} \frac{d y}{1-y} \frac{d x}{1-x}+\frac{d(x y)}{1-x y}\left(\frac{d y}{1-y}+\frac{d x}{x(x-1)}\right) .
\end{aligned}
$$

When $n=3$ let $\mathbf{x}=(x, y, z)$. Then

$$
\begin{aligned}
& \mathbf{x}(0,0,1)=z, \quad \mathbf{x}(0,1,0)=y z, \quad \mathbf{x}(1,0,0)=x y z, \quad \mathbf{x}(0,1,1)=(y, z), \\
& \mathbf{x}(1,0,1)=(x y, z), \quad \mathbf{x}(1,1,0)=(x, y z), \quad \mathbf{x}(1,1,1)=(x, y, z) .
\end{aligned}
$$

Thus

$$
\begin{aligned}
L i_{1,1,1}(x, y, z) & =\int_{(0,0,0)}^{(x, y, z)} \frac{d z}{1-z} \frac{d y}{1-y} \frac{d x}{1-x}+\frac{d(y z)}{1-y z}\left(\frac{d z}{1-z}+\frac{d y}{y(y-1)}\right) \frac{d x}{1-x} \\
& +\frac{d(y z)}{1-y z} \frac{d x}{1-x}\left(\frac{d z}{1-z}+\frac{d y}{y(y-1)}\right)+\frac{d z}{1-z} \frac{d(x y)}{1-x y}\left(\frac{d y}{1-y}+\frac{d x}{x(x-1)}\right) \\
& +\frac{d(x y z)}{1-x y z}\left(\frac{d z}{1-z}+\frac{d(x y)}{x y(x y-1)}\right)\left(\frac{d y}{1-y}+\frac{d x}{x(x-1)}\right) \\
& +\frac{d(x y z)}{1-x y z}\left(\frac{d(y z)}{1-y z}+\frac{d x}{x(x-1)}\right)\left(\frac{d z}{1-z}+\frac{d y}{y(y-1)}\right) .
\end{aligned}
$$

Lemma 4.3. The iterated path integral inside the limit of (9) depends only on the homotopy class of the path from $\boldsymbol{\varepsilon}_{n}$ to $\mathbf{x}$.

Proof. We use induction on the weight $K$ and Lemma 2.2 to prove this lemma.

When $K=1$ this is trivial. When $K=2$ there are two possibilities: the dilogarithm $L i_{2}(x)$ and the double logarithm $L i_{1,1}\left(x_{1}, x_{2}\right)$. First

$$
\operatorname{Li}_{2}(z)=\int_{0}^{z} \frac{d x}{1-x} \frac{d x}{x}
$$

and thus $\frac{d x}{1-x} \wedge \frac{d x}{x}=0$ over $\mathbb{C}$. Second, for the double logarithm $L i_{1,1}(x, y)$ as given in Example 4.2 we clearly have

$$
\frac{d y}{1-y} \wedge \frac{d x}{1-x}+\frac{d(x y)}{1-x y} \wedge\left(\frac{d y}{1-y}-\frac{d x}{1-x}-\frac{d x}{x}\right)=0 .
$$


Suppose now $K \geq 3$ and the lemma is proved for up to $K-1$. Then it is not hard to see that we only need to show that the sum of the wedge products of the last two 1-forms is zero. Let us look at the 2-form $d x_{s} \wedge d x_{t}$ for $1 \leq s \neq t \leq n$. If $|s-t|>1$ then by the symmetry of the equations (5) and (6) with respect to $s$ and $t$ and skewsymmetry of the wedge product we get the desired result. So we may assume that $|t-s|=1$.

(i) If $m_{s}>1$ and $m_{t}>1$ then without loss of generality we may assume that $t=s+1$. We have

$$
\begin{gathered}
d_{s} L i_{m_{1}, \ldots, m_{n}}(\mathbf{x})=L i_{m_{1}, \ldots, m_{s-1}, m_{s}-1, m_{s+1}, \ldots, m_{n}}(\mathbf{x}) \frac{d x_{s}}{x_{s}} \\
d_{s+1} L i_{m_{1}, \ldots, m_{n}}(\mathbf{x})=L i_{m_{1}, \ldots, m_{s}, m_{s+1}-1, m_{s+2}, \ldots, m_{n}}(\mathbf{x}) \frac{d x_{s+1}}{x_{s+1}} .
\end{gathered}
$$

Hence, by skewsymmetry of the wedge product, the sum of $\left(d x_{s} \wedge d x_{s+1}\right)$-terms cancel with the sum of $\left(d x_{s+1} \wedge d x_{s}\right)$-terms.

(ii) If $m_{s}=1$ and $m_{t}>1$ then we have (12) and (6) with $t$ replaced by $s$ and get

$$
\frac{d x_{s}}{x_{s}\left(x_{s}-1\right)} \wedge \frac{d x_{t}}{x_{t}}+\frac{d\left(x_{s} x_{t}\right)}{x_{s} x_{t}} \wedge \frac{d x_{s}}{x_{s}\left(x_{s}-1\right)}+\frac{d x_{s}}{1-x_{s}} \wedge \frac{d x_{t}}{x_{t}}+\frac{d x_{t}}{x_{t}} \wedge \frac{d x_{s}}{1-x_{s}}=0 .
$$

Here if $s=n$ then the first two terms do not occur.

(iii) If $m_{s}=m_{t}=1$ then we may assume that $t=s+1$. Take (6) with $t$ replaced by $s$ and $s+1$ respectively. Quickly we find that the sum of $\left(d x_{s} \wedge d x_{s+1}\right)$-terms is

$$
\begin{aligned}
& \frac{d x_{s+1}}{1-x_{s+1}} \wedge \frac{d x_{s}}{1-x_{s}}+\frac{d x_{s+1}}{x_{s+1}\left(x_{s+1}-1\right)} \wedge \frac{d x_{s}}{1-x_{s}} \\
& \quad+\frac{d\left(x_{s} x_{s+1}\right)}{x_{s} x_{s+1}\left(x_{s} x_{s+1}-1\right)} \wedge \frac{d x_{s}}{x_{s}\left(x_{s}-1\right)}+\frac{d\left(x_{s} x_{s+1}\right)}{1-x_{s} x_{s+1}} \wedge \frac{d x_{s}}{x_{s}\left(x_{s}-1\right)}=\frac{d x_{s+1}}{x_{s+1}} \wedge \frac{d x_{s}}{x_{s}}
\end{aligned}
$$

whereas by symmetry the sum of $\left(d x_{s+1} \wedge d x_{s}\right)$-terms is

$$
\frac{d x_{s}}{x_{s}} \wedge \frac{d x_{s+1}}{x_{s+1}}
$$

which cancels with the sum of the $\left(d x_{s} \wedge d x_{s+1}\right)$-terms.

By induction the lemma now follows from Lemma 2.2.

We now show that the multiple polylogarithms have trivial monodromy about each $x_{j}=0$, $j=1, \cdots, n$, and therefore they are actually well-defined on $S_{n}$.

Theorem 4.4. Let $p(\varepsilon)$ be a path in from $\varepsilon_{n} \in D_{n}$ to an arbitrary $\mathbf{x} \in S_{n}^{\prime}$. Let $q(\varepsilon)$ be a loop in $S_{n}^{\prime}$ based at $\varepsilon_{n} \in D_{n}$ around any $\mathcal{D}_{j 0}=\left\{x_{j}=0\right\}(j=1, \cdots, n)$ but no other irreducible components of $X_{n}^{\prime}$, then

$$
\lim _{\varepsilon \rightarrow 0}\left(\int_{q(\varepsilon) p(\varepsilon)}-\int_{p(\varepsilon)}\right) \sum_{\substack{\left(\delta_{1}, \ldots, \delta_{K}\right) \in \mathfrak{S}_{K} \\ \overrightarrow{\mathbf{J}} \in \mathfrak{S}^{K}\left(m_{1}, \ldots, m_{n}\right)}} \bigsqcup_{r=1}^{K} w_{\overrightarrow{\mathbf{j}}}^{r, \delta_{r}}\left(\mathbf{x}\left(T_{\delta_{r+1}}^{r+1} \circ \cdots \circ T_{\delta_{K}}^{K}\left(\mathbf{j}_{r}\right)\right)\right)=0 .
$$

Therefore the multiple polylogarithm $L i_{m_{1}, \ldots, m_{n}}(\mathbf{x})$ is a multi-valued holomorphic function on $S_{n}$ and can be expressed by

$$
L i_{m_{1}, \ldots, m_{n}}(\mathbf{x})=\int_{\mathbf{0}}^{\mathbf{x}} \sum_{\substack{\left(\delta_{1}, \ldots, \delta_{K}\right) \in \mathfrak{S}_{K} \\ \overrightarrow{\mathbf{J}} \in \mathfrak{S}^{K}\left(m_{1}, \ldots, m_{n}\right)}} \bigsqcup_{r=1}^{K} w_{\overrightarrow{\mathbf{J}}}^{r, \delta_{r}}\left(\mathbf{x}\left(T_{\delta_{r+1}}^{r+1} \circ \cdots \circ T_{\delta_{K}}^{K}\left(\mathbf{j}_{r}\right)\right)\right) .
$$


Proof. We prove (13) in the lemma by induction on $K$. If $K=1$ clearly $d x_{1} /\left(1-x_{1}\right)$ has no singularity at $\mathcal{D}_{10}=\left\{x_{1}=0\right\}$ and (14) is obvious. Assume the cases up to $K-1$ are true. Consequently, if $m_{1}+\cdots+m_{n}=K-1$ then $L i_{m_{1}, \ldots, m_{n}}(\mathbf{x})$ is well defined by (14) and is equal to 0 if any $x_{i}=0$ and the path from $\mathbf{0}$ to $\mathbf{x}$ does not enclose any irreducible component of $X_{n}$.

We first prove that

$$
\lim _{\varepsilon \rightarrow 0} \int_{q(\varepsilon)} \sum_{\substack{\left(\delta_{1}, \ldots, \delta_{K}\right) \in \mathfrak{S}_{K} \\ \overrightarrow{\mathbf{J}}_{\mathfrak{s}} \in \mathfrak{S}^{K}\left(m_{1}, \ldots, m_{n}\right)}} \bigsqcup_{r=1}^{K} w_{\overrightarrow{\mathbf{J}}}^{r, \delta_{r}}\left(\mathbf{x}\left(T_{\delta_{r+1}}^{r+1} \circ \cdots \circ T_{\delta_{K}}^{K}\left(\mathbf{j}_{r}\right)\right)\right)=0 .
$$

This is a special case of $(13)$ when $p(\varepsilon)$ shrinks to a point.

By equation (7) in the proof in Proposition 4.1 we find that the left hand side of (15) is equal to

$$
\lim _{\varepsilon \rightarrow 0} \int_{q(\varepsilon)} \sum_{\delta_{K}=0,1} \sum_{\substack{\left|\mathbf{j}_{K}-1\right|=K-1, \mathbf{j}_{K-1} \in \mathfrak{S}\left(m_{1}, \ldots, m_{n}\right)}} L i_{T_{\delta_{K}}^{K}\left(\mathbf{j}_{K-1}\right)}\left(\mathbf{x}\left(T_{\delta_{K}}^{K}\left(\mathbf{j}_{K-1}\right)\right)\right) w_{\overrightarrow{\mathbf{j}}}^{K, \delta_{K}}(\mathbf{x}) .
$$

By Lemma 4.3 we may assume that the path $q(\varepsilon)$ lies in the (real) two dimensional space $\cap_{i \neq j}\left\{x_{i}=\varepsilon\right\}$ enclosing $\mathcal{D}_{j 0}$ clockwise only once. By induction the terms with $\mathbf{j}_{K}-\mathbf{j}_{K-1} \neq \mathbf{u}_{j}$ in the above sum are clearly zero. Thus the integral is reduced to

$$
\left.\lim _{\varepsilon \rightarrow 0} \int_{q(\varepsilon)} \sum_{\delta_{K}=0,1} L i_{T_{\delta_{K}}^{K}\left(\mathbf{j}_{K-1}\right)}\left(\mathbf{x}\left(T_{\delta_{K}}^{K}\left(\mathbf{j}_{K-1}\right)\right)\right) w_{\overrightarrow{\mathbf{j}}}^{K, \delta_{K}}(\mathbf{x})\right), \quad \mathbf{j}_{K-1}=\mathbf{1}_{K}-\mathbf{u}_{j}
$$

since $\mathbf{j}_{K}=\mathbf{1}_{K}$. By the induction assumption the functions in front of $w_{\overrightarrow{\mathbf{J}}}^{K, \delta_{K}}(\mathbf{x})$ are regular along $\mathcal{D}_{j 0}$. If $w_{\overrightarrow{\mathbf{J}}}^{K, \delta_{K}}(\mathbf{x})$ does not have singularity along $\mathcal{D}_{j 0}$ then clearly (16) is equal to 0 . If it has singularity along $\mathcal{D}_{j 0}$ then (16) is the limit of $\pm 2 \pi i L i_{T_{\delta_{K}}^{K}\left(\mathbf{j}_{K-1}\right)}\left(\mathbf{x}\left(T_{\delta_{K}}^{K}\left(\mathbf{j}_{K-1}\right)\right)\right)$ evaluated at $(\epsilon, \ldots, \epsilon, 0, \epsilon, \ldots, \epsilon)$ where 0 is at the $j$ th place as $\varepsilon \rightarrow 0$ because $q(\varepsilon)$ lies in $\cap_{i \neq j}\left\{x_{i}=\varepsilon\right\}$ and does not enclose any irreducible component of $X_{n}$. This limit is equal to 0 by the induction assumption.

For brevity we drop the limit and $\varepsilon$ in the rest of the proof. From Lemma 2.1(iii)

$$
\begin{aligned}
& \left(\int_{q p}-\int_{p}\right) \sum_{\substack{\left(\delta_{1}, \ldots, \delta_{K}\right) \in \mathfrak{S}_{K} \\
\overrightarrow{\mathbf{J}} \in \mathfrak{S}^{K}\left(m_{1}, \ldots, m_{n}\right)}} \bigsqcup_{r=1}^{K} w_{\overrightarrow{\mathbf{J}}}^{r, \delta_{r}}\left(\mathbf{x}\left(T_{\delta_{r+1}}^{r+1} \circ \cdots \circ T_{\delta_{K}}^{K}\left(\mathbf{j}_{r}\right)\right)\right) \\
& =\sum_{s=1}^{K} \int_{\substack { q \\
\begin{subarray}{c}{\left(\delta_{1}, \ldots, \delta_{K}\right) \in \mathfrak{S}_{K} \\
\mathbf{J}_{1} \in \mathfrak{S}^{K}\left(m_{1}, \ldots, m_{n}\right){ q \\
\begin{subarray} { c } { ( \delta _ { 1 } , \ldots , \delta _ { K } ) \in \mathfrak { S } _ { K } \\
\mathbf { J } _ { 1 } \in \mathfrak { S } ^ { K } ( m _ { 1 } , \ldots , m _ { n } ) } }\end{subarray}} \bigsqcup_{r=1}^{s} w_{\overrightarrow{\mathbf{J}}}^{r, \delta_{r}}\left(\mathbf{x}\left(T_{\delta_{r+1}}^{r+1} \circ \cdots \circ T_{\delta_{K}}^{K}\left(\mathbf{j}_{r}\right)\right)\right) \\
& \cdot \int_{p} \bigsqcup_{r=s+1}^{K} w_{\overrightarrow{\mathbf{J}}}^{r, \delta_{r}}\left(\mathbf{x}\left(T_{\delta_{r+1}}^{r+1} \circ \cdots \circ T_{\delta_{K}}^{K}\left(\mathbf{j}_{r}\right)\right)\right) .
\end{aligned}
$$

We want to show that for each fixed $s$ the inner sum is zero. The formula (15) shows this is true for $s=K$. When $s=K-1$ we can divide the sum over products like

$$
\int_{q} \phi_{1} \cdots \phi_{K-1} \int_{p} \phi_{K}
$$

into sub-sums each one of which is produced by grouping all terms with $\phi_{K}=w_{\overrightarrow{\mathbf{J}}}^{K, \delta_{K}}(\mathbf{x})$ for some fixed $\delta_{K}$ and $\mathbf{j}_{K-1}$ which means that $\phi_{K}$ is fixed. We see that the iterated integral of 
every sub-sum is 0 by using equation (7) and applying (15) with $K$ replaced by $K-1$ and $\mathbf{x}$ replaced by $\mathbf{x}\left(T_{\delta_{K}}^{K}\left(\mathbf{j}_{K-1}\right)\right)$.

For any $1 \leq s \leq n-2$ the sum over products like

$$
\int_{q} \phi_{1} \cdots \phi_{s} \int_{p} \phi_{s+1} \cdots \phi_{K}
$$

can be treated similarly by fixing $\phi_{s+1} \ldots \phi_{K}$ first and then applying (15) with $K=s$ and $\mathbf{x}$ replaced by $\mathbf{x}\left(T_{\delta_{s+1}}^{s+1} \circ \cdots \circ T_{\delta_{K}}^{K}\left(\mathbf{j}_{s}\right)\right)$.

This completes the proof of the theorem.

\section{Multiple logarithms}

To study the mixed Hodge structure associated with the multiple polylogarithms it is imperative that we resolve the monodromy of them. In this section we carry this out for multiple $\operatorname{logarithm} \mathfrak{L}_{n}\left(x_{1}, \ldots, x_{n}\right)$. We first provide a cleaner form of its analytic continuation.

Keeping the notation in the previous sections we have $\mathfrak{S}(1, \ldots, 1)=\mathfrak{S}_{n}$ and $K=n$ for multiple logarithms. Though we can get the analytic continuation of the multiple logarithms by (9) immediately, we actually have a cleaner expression in this special case.

For any $\mathbf{i}=\left(i_{1}, \ldots, i_{n}\right) \in \mathfrak{S}_{n}$ with $i_{s}=0$ we define

$$
\operatorname{pos}\left(\mathbf{i}, \mathbf{i}+\mathbf{u}_{s}\right)=s
$$

as the position where the component is increased by 1 . For example $\operatorname{pos}((1,0),(1,1))=2$. We define the position functions $f_{n}^{1}, \ldots, f_{n}^{n}$ on $\overrightarrow{\mathbf{J}} \in \mathfrak{S}_{n}^{n}$ as follows:

$$
f_{n}^{1}(\overrightarrow{\mathbf{J}})=1, \quad f_{n}^{t}(\overrightarrow{\mathbf{J}})=\operatorname{pos}\left(\mathbf{j}_{t-1}, \mathbf{j}_{t}\right), \text { for } 2 \leq t \leq n .
$$

These functions tell us the places where the increments occur in the queue of $\overrightarrow{\mathbf{J}}$. Let

$$
w_{1}(\mathbf{x}):=d \log \left(\frac{1}{1-x_{1}}\right) ; \quad w_{t}(\mathbf{x}):=d \log \left(\frac{1-x_{t-1}^{-1}}{1-x_{t}}\right), \text { for } 2 \leq t \leq n .
$$

Proposition 5.1. The multiple logarithm $\mathfrak{L}_{n}(\mathbf{x})$ is a multi-valued holomorphic function on $S_{n}$ and can be expressed by

$$
\mathfrak{L}_{n}(\mathbf{x})=\sum_{\overrightarrow{\mathbf{J}}=\left(\mathbf{j}_{1}, \ldots, \mathbf{j}_{n}\right) \in \mathfrak{S}_{n}^{n}} \int_{\mathbf{0}}^{\mathbf{x}} w_{f_{n}^{1}(\overrightarrow{\mathbf{J}})}\left(\mathbf{x}\left(\mathbf{j}_{1}\right)\right) w_{f_{n}^{2}(\overrightarrow{\mathbf{J}})}\left(\mathbf{x}\left(\mathbf{j}_{2}\right)\right) \cdots w_{f_{n}^{n}(\overrightarrow{\mathbf{J}})}\left(\mathbf{x}\left(\mathbf{j}_{n}\right)\right)
$$

Proof. Similar to the proof of Proposition 4.1 and Theorem 4.4.

We now turn to the monodromy of multiple logarithms.

Lemma 5.2. Let $p$ be a path from $\mathbf{0}$ to $\mathbf{x}$ in $S_{n}$. Let $q \in \pi_{1}\left(S_{n}, \mathbf{x}\right)$ be a loop turning around the component $\mathcal{D}_{n n}=\left\{x_{n}-1=0\right\}$ only once but no other irreducible components of $X_{n}$ such that $\int_{q} d \log \left(1-x_{n}\right)=-2 \pi i$. Then

$$
(\Theta(q)-\mathrm{id}) \mathfrak{L}_{n}(\mathbf{x})=-2 \pi i \mathfrak{L}_{n-1}\left(x_{1}, \ldots, x_{n-1}\right) .
$$

Proof. By Lemma 2.2 we may assume that $q$ is based at $\mathbf{0}$ instead of $\mathbf{x}$. We begin by moving the base of $p$ and $q$ to $\varepsilon_{n}$ near $\mathbf{0}$ and later we take the limit $\boldsymbol{\varepsilon}_{n} \rightarrow \mathbf{0}$.

Let $p(\varepsilon)$ and $q(\varepsilon)$ be the corresponding loop based at $\varepsilon_{n}=(\varepsilon, \cdots, \varepsilon) \in D_{n}$. By Lemma 4.3 we can take the loop $q(\varepsilon)$ in the two dimensional plane (over $\mathbb{R}$ ) $x_{1}=\cdots=x_{n-1}=\varepsilon$ 
counterclockwise. By Lemma 2.1(ii)

$$
\begin{aligned}
&\left(\int_{q(\varepsilon) p(\varepsilon)}-\int_{p(\varepsilon)}\right) \sum_{\overrightarrow{\mathbf{J}}=\left(\mathbf{j}_{1}, \ldots, \mathbf{j}_{n}\right) \in \mathfrak{S}_{n}^{n}} w_{f_{n}^{1}(\overrightarrow{\mathbf{J}})}\left(\mathbf{x}\left(\mathbf{j}_{1}\right)\right) w_{f_{n}^{2}(\overrightarrow{\mathbf{J}})}\left(\mathbf{x}\left(\mathbf{j}_{2}\right)\right) \cdots w_{f_{n}^{n}(\overrightarrow{\mathbf{J}})}\left(\mathbf{x}\left(\mathbf{j}_{n}\right)\right) \\
&= \sum_{\overrightarrow{\mathbf{J}}=\left(\mathbf{j}_{1}, \ldots, \mathbf{j}_{n}\right) \in \mathfrak{S}_{n}^{n}} \sum_{t=1}^{n} \int_{q(\varepsilon)} w_{f_{n}^{1}(\overrightarrow{\mathbf{J}})}\left(\mathbf{x}\left(\mathbf{j}_{1}\right)\right) \cdots w_{f_{n}^{t}(\overrightarrow{\mathbf{J}})}\left(\mathbf{x}\left(\mathbf{j}_{t}\right)\right) \\
& \cdot \int_{p(\varepsilon)} w_{f_{n}^{t+1}(\overrightarrow{\mathbf{J}})}\left(\mathbf{x}\left(\mathbf{j}_{t+1}\right)\right) \cdots w_{f_{n}^{n}(\overrightarrow{\mathbf{J}})}\left(\mathbf{x}\left(\mathbf{j}_{n}\right)\right) .
\end{aligned}
$$

If $\mathbf{j}_{1}=\mathbf{u}_{s}$ for some $1 \leq s \leq n-1$ then

$$
w_{1}\left(\mathbf{x}\left(\mathbf{j}_{1}\right)\right)=\frac{d\left(x_{s} \ldots x_{n}\right)}{1-x_{s} \ldots x_{n}} .
$$

Thus for any $t=2, \cdots, n$

$$
\begin{aligned}
& \int_{q(\varepsilon)} w_{f_{n}^{1}(\overrightarrow{\mathbf{J}})}\left(\mathbf{x}\left(\mathbf{j}_{1}\right)\right) \cdots w_{f_{n}^{t}(\overrightarrow{\mathbf{J}})}\left(\mathbf{x}\left(\mathbf{j}_{t}\right)\right) \\
&=\int_{q(\varepsilon)}-\log \left(\frac{1-\varepsilon^{n-s} x_{n}}{1-\varepsilon^{n-s+1}}\right) w_{f_{n}^{2}(\overrightarrow{\mathbf{J}})}\left(\mathbf{x}\left(\mathbf{j}_{2}\right)\right) \cdots w_{f_{n}^{t}(\overrightarrow{\mathbf{J}})}\left(\mathbf{x}\left(\mathbf{j}_{t}\right)\right) \rightarrow 0 \text { as } \varepsilon \rightarrow 0 .
\end{aligned}
$$

We now only need to look at those $\overrightarrow{\mathbf{J}} \in \mathfrak{S}_{n}^{n}$ with $\mathbf{j}_{1}=(0, \ldots, 0,1)$. Then the last component of $\mathbf{j}_{t}$ is always 1 for $t=2, \ldots, n$. Thus the variable $\mathbf{x}\left(\mathbf{j}_{t}\right)=\left(\ldots, x_{n}\right)$ has $t$ components and $f_{n}^{t}(\mathbf{j}) \leq t-1$ because 0 cannot appear at the last position of $\rho_{\mathbf{j}_{t}}\left(\mathbf{j}_{t-1}\right)$. Therefore, $w_{f_{n}^{t}(\overrightarrow{\mathbf{J}})}\left(\mathbf{x}\left(\mathbf{j}_{t}\right)\right)$ does not involve the variable $x_{n}$ for $t=2, \ldots, n$.

Suppose now $\phi_{2}, \ldots, \phi_{n}$ are 1-forms on $S_{n}$ that do not involve $x_{n}$. Then by Lemma 2.1(ii)

$$
\begin{aligned}
& \int_{q(\varepsilon) p(\varepsilon)} \frac{d x_{n}}{1-x_{n}} \phi_{2} \cdots \phi_{n}-\int_{p(\varepsilon)} \frac{d x_{n}}{1-x_{n}} \phi_{2} \cdots \phi_{n} \\
= & \int_{q(\varepsilon)} \frac{d x_{n}}{1-x_{n}} \int_{p(\varepsilon)} \phi_{2} \cdots \phi_{n}+\sum_{t=2}^{n} \int_{q(\varepsilon)} \frac{d x_{n}}{1-x_{n}} \phi_{2} \cdots \phi_{t} \int_{p(\varepsilon)} \phi_{t+1} \cdots \phi_{n} \\
= & -2 \pi i \int_{p(\varepsilon)} \phi_{2} \cdots \phi_{n}+\sum_{t=2}^{n}(-1)^{t+1} \int_{q(\varepsilon)^{-1}} \phi_{t} \cdots \phi_{2} \frac{d x_{n}}{1-x_{n}} \int_{p(\varepsilon)} \phi_{t+1} \cdots \phi_{n} .
\end{aligned}
$$

But none of $\phi_{t}$ for $t=2, \cdots, n$, involves variable $x_{n}$ by assumption and therefore the inner most integral

$$
\int_{(\varepsilon, \cdots, \varepsilon, \varepsilon)}^{\left(\varepsilon, \cdots, \varepsilon, x_{n}\right)} \phi_{t}=0
$$

for any $x_{n} \in S_{n}$. Hence

$$
\lim _{\varepsilon \rightarrow 0}\left(\int_{q(\varepsilon) p(\varepsilon)}-\int_{p(\varepsilon)}\right) \frac{d x_{n}}{1-x_{n}} \phi_{2} \cdots \phi_{n}=-2 \pi i \int_{p} \phi_{2} \cdots \phi_{n} .
$$

The lemma now follows from the one-to-one correspondence

$$
\mathfrak{S}_{n-1}^{n-1} \longleftrightarrow\left\{\overrightarrow{\mathbf{J}} \in \mathfrak{S}_{n}^{n}: \mathbf{j}_{1}=\mathbf{u}_{1}\right\}
$$

Theorem 5.3. Let $1 \leq s \leq n$. Let $p$ be a path from $\mathbf{0}$ to $\mathbf{x}$ in $S_{n}$. Let $q_{s} \in \pi_{1}\left(S_{n}, \mathbf{x}\right)$ enclose the component $\mathcal{D}_{s n}=\left\{x_{s} \cdots x_{n}=1\right\}$ only once in $S_{n}$ but no other irreducible components of $X_{n}$ such that $\int_{q_{s}} d \log \left(1-x_{s} \cdots x_{n}\right)=-2 \pi i$. Then

$$
\left(\Theta\left(q_{s}\right)-\mathrm{id}\right) \mathfrak{L}_{n}(\mathbf{x})=-2 \pi i \mathfrak{L}_{s-1}\left(x_{1}, \ldots, x_{s-1}\right) \cdot \mathfrak{L}_{n-s}(\mathbf{y}(s))
$$


where

$$
\mathbf{y}(s)=\left(\frac{1-x_{s} x_{s+1}}{1-x_{s}}, \ldots, \frac{1-x_{s} \ldots x_{n}}{1-x_{s} \ldots x_{n-1}}\right) .
$$

Proof. The case $s=n$ is proved by Lemma 5.2. We now prove the case $s=1$. The general case will follow from these two cases by shuffle relations.

By Lemma 4.3 we may assume that the path $q$ lie entirely in the (real) 2-dimensional plane $x_{1}=x_{1}, \ldots, x_{n-1}=x_{n-1}$. Suppose $s=1$ and $\mathbf{y}=\mathbf{y}(1)=\left(y_{1}, \ldots, y_{n-1}\right)$. Let $\tilde{\mathfrak{S}}_{n}=\left\{\mathbf{j}=\left(j_{1}, \ldots, j_{n}\right) \in \mathfrak{S}_{n}: j_{1}=1\right\}$ and

$$
\tilde{\mathfrak{S}}_{n}^{n}=\left\{\left(\mathbf{j}_{1}, \ldots, \mathbf{j}_{n}\right) \in \mathfrak{S}_{n}^{n}: \mathbf{j}_{t} \in \tilde{\mathfrak{S}}_{n} \text { for } t=1, \ldots, n\right\} .
$$

Then we can quickly find as in the proof of Lemma 5.2 that

$$
\begin{aligned}
& \left(\int_{p q}-\int_{p} \sum_{\overrightarrow{\mathbf{J}}=\left(\mathbf{j}_{1}, \ldots, \mathbf{j}_{n}\right) \in \mathfrak{S}_{n}^{n}} w_{f_{n}^{1}(\overrightarrow{\mathbf{J}})}\left(\mathbf{x}\left(\mathbf{j}_{1}\right)\right) w_{f_{n}^{2}(\overrightarrow{\mathbf{J}})}\left(\mathbf{x}\left(\mathbf{j}_{2}\right)\right) \cdots w_{f_{n}^{n}(\overrightarrow{\mathbf{J}})}\left(\mathbf{x}\left(\mathbf{j}_{n}\right)\right)\right. \\
& =\int_{q \overrightarrow{\mathbf{J}}=\left(\mathbf{j}_{1}, \ldots, \mathbf{j}_{n}\right) \in \tilde{\mathfrak{S}}_{n}^{n}} w_{f_{n}^{1}(\overrightarrow{\mathbf{J}})}\left(\mathbf{x}\left(\mathbf{j}_{1}\right)\right) w_{f_{n}^{2}(\overrightarrow{\mathbf{J}})}\left(\mathbf{x}\left(\mathbf{j}_{2}\right)\right) \cdots w_{f_{n}^{n}(\overrightarrow{\mathbf{J}})}\left(\mathbf{x}\left(\mathbf{j}_{n}\right)\right) \\
& =(-1)^{n} \sum_{\overrightarrow{\mathbf{J}}=\left(\mathbf{j}_{1}, \ldots, \mathbf{j}_{n}\right) \in \tilde{\mathfrak{S}}_{n}^{n}} \int_{q^{-1}} w_{f_{n}^{n}(\overrightarrow{\mathbf{J}})}\left(\mathbf{x}\left(\mathbf{j}_{n}\right)\right) \cdots w_{f_{n}^{2}(\overrightarrow{\mathbf{J}})}\left(\mathbf{x}\left(\mathbf{j}_{2}\right)\right) w_{f_{n}^{1}(\overrightarrow{\mathbf{J}})}\left(\mathbf{x}\left(\mathbf{j}_{1}\right)\right) \\
& =-2 \pi i \sum_{\overrightarrow{\mathbf{J}}=\left(\mathbf{j}_{1}, \ldots, \mathbf{j}_{n}\right) \in \tilde{\mathfrak{S}}_{n}^{n}} \int_{\left(x_{1}, \ldots, x_{n-1},\left(x_{1} \ldots x_{n-1}\right)^{-1}\right)}^{\left(x_{1}, \ldots, x_{n-1}, x_{n}\right)} w_{f_{n}^{2}(\overrightarrow{\mathbf{J}})}\left(\mathbf{x}\left(\mathbf{j}_{2}\right)\right) \cdots w_{f_{n}^{n}(\overrightarrow{\mathbf{J}})}\left(\mathbf{x}\left(\mathbf{j}_{n}\right)\right)
\end{aligned}
$$

by Lemma 2.1(iii). Here the path in the integral inside the last sum is a contractible path in $S_{n}$. Define

$$
\begin{aligned}
\mu: \mathfrak{S}_{n} & \longrightarrow \mathfrak{S}_{n-1} \\
\left(i_{1}, \ldots, i_{n}\right) & \longmapsto\left(i_{2}, \ldots, i_{n}\right)
\end{aligned}
$$

and extend it to $\mathfrak{S}_{n}^{n}$ by mapping $\left(\mathbf{i}_{1}, \ldots, \mathbf{i}_{n}\right)$ to $\left(\mu\left(\mathbf{i}_{2}\right), \ldots, \mu\left(\mathbf{i}_{n}\right)\right)$. This clearly induces a bijection $\tilde{\mathfrak{S}}_{n}^{n} \leftrightarrow \mathfrak{S}_{n-1}^{n-1}$. Now the case $s=1$ immediately follows from the following claim and the fact that $\mathfrak{L}_{n-1}(\mathbf{y})$ evaluated at $\left(x_{1}, \ldots, x_{n-1},\left(x_{1} \ldots x_{n-1}\right)^{-1}\right)$ is 0 .

Claim. For $2 \leq r \leq s$

$$
w_{r}\left(\mathbf{x}\left(\mathbf{j}_{s}\right)\right)=w_{r-1}\left(\mathbf{y}\left(\mu\left(\mathbf{j}_{s}\right)\right)\right)
$$

Proof of the Claim. Let $\mathbf{j}_{s}=\sum_{i=1}^{s} \mathbf{u}_{t_{i}}$ where $1=t_{1}<\cdots<t_{s} \leq n$. Then $\mu\left(\mathbf{j}_{s}\right)=\sum_{i=2}^{s} \mathbf{u}_{t_{i}-1}$ in $\mathfrak{S}_{n-1}$ and

$$
\begin{aligned}
\mathbf{x}\left(\mathbf{j}_{s}\right) & =\left(x_{1} \cdots x_{t_{2}-1}, x_{t_{2}} \cdots x_{t_{3}-1}, \ldots, x_{t_{s}} \cdots x_{n}\right) \\
\mathbf{y}\left(\mu\left(\mathbf{j}_{s}\right)\right) & =\left(y_{t_{2}-1} \cdots y_{t_{3}-2}, y_{t_{3}-1} \cdots y_{t_{4}-2}, \ldots, y_{t_{s}-1} \cdots y_{n-1}\right) .
\end{aligned}
$$

When $r=2$ we have

$$
w_{1}\left(\mathbf{y}\left(\mu\left(\mathbf{j}_{s}\right)\right)\right)=w_{1}\left(y_{t_{2}-1} \cdots y_{t_{3}-2}\right)=w_{1}\left(\frac{1-x_{1} \cdots x_{t_{3}-1}}{1-x_{1} \cdots x_{t_{2}-1}}\right)=w_{2}\left(\mathbf{x}\left(\mathbf{j}_{s}\right)\right)
$$

by the obvious identity

$$
w_{2}(X, Y)=w_{1}\left(\frac{1-X Y}{1-X}\right) .
$$

By the same identity, when $r>2$ we have

$$
w_{r}\left(\mathbf{x}\left(\mathbf{j}_{s}\right)\right)=w_{2}\left(x_{t_{r-1}} \cdots x_{t_{r}-1}, x_{t_{r}} \cdots x_{t_{r+1}-1}\right)=w_{1}\left(\frac{1-x_{t_{r-1}} \cdots x_{t_{r+1}-1}}{1-x_{t_{r-1}} \cdots x_{t_{r}-1}}\right) .
$$


On the other hand

$$
\begin{aligned}
w_{r-1}\left(\mathbf{y}\left(\mu\left(\mathbf{j}_{s}\right)\right)\right) & =w_{2}\left(y_{t_{r-1}-1} \cdots y_{t_{r}-2}, y_{t_{r}-1} \cdots y_{t_{r+1}-2}\right) \\
& =w_{2}\left(\frac{1-x_{1} \cdots x_{t_{r}-1}}{1-x_{1} \cdots x_{t_{r-1}-1}}, \frac{1-x_{1} \cdots x_{t_{r+1}-1}}{1-x_{1} \cdots x_{t_{r}-1}}\right) \\
\text { (by (18) again) } & =w_{1}\left(\left(1-\frac{1-x_{1} \cdots x_{t_{r+1}-1}}{1-x_{1} \cdots x_{t_{r-1}-1}}\right) /\left(1-\frac{1-x_{1} \cdots x_{t_{r}-1}}{1-x_{1} \cdots x_{t_{r-1}-1}}\right)\right. \\
& =w_{1}\left(\frac{x_{1} \cdots x_{t_{r+1}-1}-x_{1} \cdots x_{t_{r-1}-1}}{x_{1} \cdots x_{t_{r}-1}-x_{1} \cdots x_{t_{r-1}-1}}\right) \\
& =w_{r}\left(\mathbf{x}\left(\mathbf{j}_{s}\right)\right) .
\end{aligned}
$$

The claim now is proved.

Let $2 \leq s \leq n-1$ and $\mathbf{y}=\mathbf{y}(s)$. By Lemma 4.3 we can move our base point of the loop $q_{s}$ from $\mathbf{x}$ to $\varepsilon_{n}$ close to $\mathbf{0}$. Let $q(\varepsilon)$ be a loop around $\mathcal{D}_{s n}$ based at $\varepsilon_{n}$ lying entirely in $x_{1}=\cdots x_{n-1}=\varepsilon$ such that $\int_{q(\varepsilon)} d \log \left(1-x_{s} \cdots x_{n}\right)=-2 \pi i$. Let $p(\varepsilon)$ be a path from $\varepsilon_{n}$ to $\mathrm{x}$ in $S_{n}$. Then as in Lemma 5.2 we find that

$$
\begin{array}{r}
\lim _{\varepsilon \rightarrow 0}\left(\int_{q(\varepsilon) p(\varepsilon)}-\int_{p(\varepsilon)}\right) \sum_{\overrightarrow{\mathbf{J}}=\left(\mathbf{j}_{1}, \ldots, \mathbf{j}_{n}\right) \in \mathfrak{S}_{n}^{n}} w_{f_{n}^{1}(\overrightarrow{\mathbf{J}})}\left(\mathbf{x}\left(\mathbf{j}_{1}\right)\right) w_{f_{n}^{2}(\overrightarrow{\mathbf{J}})}\left(\mathbf{x}\left(\mathbf{j}_{2}\right)\right) \cdots w_{f_{n}^{n}(\overrightarrow{\mathbf{J}})}\left(\mathbf{x}\left(\mathbf{j}_{n}\right)\right) \\
=-2 \pi i \sum_{\overrightarrow{\mathbf{J}} \in \mathfrak{S}_{n}^{n}, \mathbf{j}_{1}=\mathbf{u}_{s}} \lim _{\varepsilon \rightarrow 0} \int_{p(\varepsilon)} w_{f_{n}^{2}(\overrightarrow{\mathbf{J}})}\left(\mathbf{x}\left(\mathbf{j}_{2}\right)\right) \cdots w_{f_{n}^{n}(\overrightarrow{\mathbf{J}})}\left(\mathbf{x}\left(\mathbf{j}_{n}\right)\right)
\end{array}
$$

For any $\mathbf{j} \in \mathfrak{S}_{n}^{n}$ with $\mathbf{j}_{1}=\mathbf{u}_{s}$ we define the type of $\mathbf{j}_{t}(t \geq 2)$ by

$$
\operatorname{Type}\left(\mathbf{j}_{t}\right)= \begin{cases}(\mathrm{I}) & \text { if } \mathbf{j}_{t}=\mathbf{j}_{t-1}+\mathbf{u}_{m} \text { and } m<s ; \\ \text { (II) } & \text { if } \mathbf{j}_{t}=\mathbf{j}_{t-1}+\mathbf{u}_{m} \text { and } m>s .\end{cases}
$$

Note that $m=f_{n}^{t}(\overrightarrow{\mathbf{J}})$. Now each term in the sum (19) has the form $\phi_{2} \cdots \phi_{n}$ with $s-1$ of them being of type (I) and $n-s$ of them being of type (II). If $\phi_{t}=w_{f_{n}^{t}(\overrightarrow{\mathbf{J}})}\left(\mathbf{x}\left(\mathbf{j}_{t}\right)\right)$ is of type (I) then $f_{n}^{t}(\overrightarrow{\mathbf{J}})<s$ and the $s$-th component of $\mathbf{j}_{t}$ is 1 which implies that $\phi_{t}$ does not involve the variables $x_{s}, \ldots, x_{n}$. Similar argument applies to type (II) 1-forms which does not involve the variables $x_{1}, \ldots, x_{s-1}$. Furthermore, altogether there are $(n-1)$ ! terms in the sum of (19) which are all different from each other. An easy computation using Lemma 5.2 for case $s=n$ and the above claim in the proof of case $s=1$ then yields that each term in the sum is a term in the expansion of the right hand side of ( $\operatorname{setting} \mathbf{x}^{\prime}=\left(x_{1}, \ldots, x_{s-1}\right)$ )

$$
\begin{aligned}
\mathfrak{L}_{s-1}\left(\mathbf{x}^{\prime}\right) \mathfrak{L}_{n-s}(\mathbf{y})= & \lim _{\varepsilon \rightarrow 0} \int_{p(\varepsilon)} \sum_{\overrightarrow{\mathbf{r}} \in \mathfrak{S}_{s-1}^{s-1}} w_{f_{s-1}^{1}(\overrightarrow{\mathbf{r}})}\left(\mathbf{x}^{\prime}\left(\mathbf{i}_{1}\right)\right) \cdots w_{f_{s-1}^{s-1}(\overrightarrow{\mathbf{r}})}\left(\mathbf{x}^{\prime}\left(\mathbf{i}_{s-1}\right)\right) \\
& \cdot \lim _{\varepsilon \rightarrow 0} \int_{p(\varepsilon)} \sum_{\overrightarrow{\mathbf{k}} \in \mathfrak{S}_{n-s}^{n-s}} w_{f_{n-s}^{1}(\overrightarrow{\mathbf{k}})}\left(\mathbf{y}\left(\mathbf{k}_{1}\right)\right) \cdots w_{f_{n-s}^{n-s}(\overrightarrow{\mathbf{k}})}\left(\mathbf{y}\left(\mathbf{k}_{n-s}\right)\right)
\end{aligned}
$$

after the shuffle relation of Lemma 2.1(iv) is applied to it. We have used the fact that in the computation of case $s=1$, we can move the base from $\mathbf{x}$ to $\varepsilon_{n}$ and then take $\varepsilon \rightarrow 0$. This provides the expression of the function $\mathfrak{L}_{n-s}(\mathbf{y})$ in the above.

Now on the right hand side of $(20)$ there are $(s-1) !(n-s) !$ terms each of which produces exactly $\left(\begin{array}{c}n-1 \\ s-1\end{array}\right)$ terms by the shuffle relation. Therefore the right hand side of (20) will produce exactly $(n-1)$ ! terms after the shuffle relation is applied.

This completes the proof of the theorem.

The next result shows that the monodromy produced by Theorem 5.3 is the only kind of monodromy of the multiple logarithms. 
Proposition 5.4. The monodromy of $\mathfrak{L}_{n}(\mathbf{x})$ about $\mathcal{D}_{i i}=\left\{x_{i}=1\right\}, 1 \leq i<n$, and $\mathcal{D}_{i j}=$ $\left\{x_{i} \cdots x_{j}=1\right\}, 1 \leq i<j<n$, is trivial.

Proof. Let $p \in \pi_{1}\left(S_{n}, \mathbf{x}\right)$ be a path from $\mathbf{0}$ to $\mathbf{x}$ and $q \in \pi_{1}\left(S_{n}, \mathbf{0}\right)$ be a loop which encloses $\mathcal{D}_{i j}$ only once but no other irreducible component of $X_{n}$.

It suffices to look at the 1 -forms in (17) which have singularities along $\mathcal{D}_{i j}, 1 \leq i \leq j<n$. Suppose $\overrightarrow{\mathbf{J}}=\left(\mathbf{j}_{1}, \ldots, \mathbf{j}_{n}\right) \in \mathfrak{S}_{n}^{n}$ and $\mathbf{j}_{s}=\left(j_{1}, \ldots, j_{n}\right)$. Because $\mathbf{j}_{s}$ has depth $s$ we may assume that $j_{t_{1}}, \ldots, j_{t_{s}}$ are the only nonzero components of $\mathbf{j}_{s}$. Let $\mathbf{y}=\mathbf{x}\left(\mathbf{j}_{s}\right)=\left(y_{1}, \ldots, y_{s}\right)$ then

$$
w_{r}(\mathbf{y})=\frac{d y_{r}}{1-y_{r}}-\frac{d y_{r-1}}{y_{r-1}}-\frac{d y_{r-1}}{1-y_{r-1}}, \quad 1 \leq r \leq s
$$

where the last two terms do not appear if $r=1$. This 1 -form has singularity along $\mathcal{D}_{i j}$, $1 \leq i \leq j<n$, if $t_{r}=i$ and $t_{r+1}=j+1$. If this is the case then

$$
w_{r+1}(\mathbf{y})=\frac{d y_{r+1}}{1-y_{r+1}}-\frac{d y_{r}}{y_{r}}-\frac{d y_{r}}{1-y_{r}}
$$

has singularity along $\mathcal{D}_{i j}$ too. These two 1 -forms correspond to the following choices of $\mathbf{j}_{s-1}$ respectively:

$$
\begin{aligned}
& \mathbf{j}_{s-1}^{\prime}=\mathbf{j}_{s}-\mathbf{u}_{i}=(\ldots, 0,1,0, \ldots, 0,0,0, \ldots, 0,1,0, \ldots, 0,1,0, \ldots) . \\
& \begin{array}{llll}
\uparrow & \uparrow & \uparrow & \uparrow
\end{array} \\
& t_{r} \text {-th } \quad i \text {-th } \quad(j+1) \text {-st } \quad t_{r+1} \text {-th }
\end{aligned}
$$

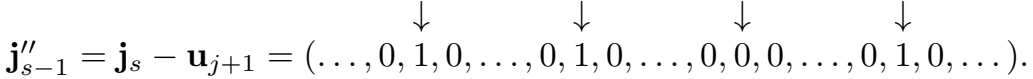

Note $j<n$ by assumption so $\mathbf{u}_{j+1}$ makes sense. Thus for each fixed $\mathbf{j}_{s}$ with $t_{r}=i$ and $t_{r+1}=j+1$ we can regroup all terms in (17) which have singularity along $\mathcal{D}_{i j}$ into sub-sums of only two terms, one for

$$
\overrightarrow{\mathbf{J}}^{\prime}=\left(\mathbf{j}_{1}, \ldots, \mathbf{j}_{s-2}, \mathbf{j}_{s-1}^{\prime}, \mathbf{j}_{s}, \mathbf{j}_{s+1}, \ldots, \mathbf{j}_{n}\right)
$$

and the other for

$$
\overrightarrow{\mathbf{J}}^{\prime \prime}=\left(\mathbf{j}_{1}, \ldots, \mathbf{j}_{s-2}, \mathbf{j}_{s-1}^{\prime \prime}, \mathbf{j}_{s}, \mathbf{j}_{s+1}, \ldots, \mathbf{j}_{n}\right)
$$

with arbitrarily fixed $\mathbf{j}_{1}, \ldots, \mathbf{j}_{s-2}, \mathbf{j}_{s+1}, \ldots, \mathbf{j}_{n}$, where we then must have $\mathbf{j}_{s-2}=\mathbf{j}_{s}-\mathbf{u}_{i}-\mathbf{u}_{j+1}$. For such two terms we have by Lemma 2.1(ii)

$$
\begin{aligned}
& (\Theta(q)-\mathrm{id}) \int_{p} \sum_{\overrightarrow{\mathbf{J}}=\overrightarrow{\mathbf{J}}^{\prime}, \overrightarrow{\mathbf{J}}^{\prime \prime}} \bigsqcup_{r=1}^{n} w_{f_{n}^{r}(\overrightarrow{\mathbf{J}})}\left(\mathbf{x}\left(\mathbf{j}_{r}\right)\right)=\int_{p_{r=s+1}} \bigsqcup_{f_{n}^{r}(\overrightarrow{\mathbf{J}})}^{n}\left(\mathbf{x}\left(\mathbf{j}_{r}\right)\right) \\
& \cdot \int_{q}^{s-2} \bigsqcup_{r=1}^{s} w_{f_{n}^{r}(\overrightarrow{\mathbf{J}})}\left(\mathbf{x}\left(\mathbf{j}_{r}\right)\right)\left[w_{f_{n}^{s-1}\left(\overrightarrow{\mathbf{J}}^{\prime}\right)}\left(\mathbf{x}\left(\mathbf{j}_{s-1}^{\prime}\right)\right) w_{r}\left(\mathbf{x}\left(\mathbf{j}_{s}\right)\right)+w_{f_{n}^{s-1}\left(\overrightarrow{\mathbf{J}}^{\prime \prime}\right)}\left(\mathbf{x}\left(\mathbf{j}_{s-1}^{\prime \prime}\right)\right) w_{r+1}\left(\mathbf{x}\left(\mathbf{j}_{s}\right)\right)\right]
\end{aligned}
$$

Here we have taken the liberty to drop the primes when it is the same to write ' or ". We see that the second iterated integral is zero because

$$
\begin{aligned}
w_{f_{n}^{s-1}\left(\overrightarrow{\mathbf{J}}^{\prime}\right)}\left(\mathbf{x}\left(\mathbf{j}_{s-1}^{\prime}\right)\right) & =w_{r}\left(\ldots, x_{t_{r-1}} \cdots x_{j}, x_{j+1} \cdots x_{t_{r+1}}, \ldots\right) \\
w_{f_{n}^{s-1}\left(\overrightarrow{\mathbf{J}}^{\prime \prime}\right)}\left(\mathbf{x}\left(\mathbf{j}_{s-1}^{\prime \prime}\right)\right) & =w_{r}\left(\ldots, x_{t_{r-1}} \cdots x_{i-1}, x_{i} \cdots x_{t_{r+1}}, \ldots\right)
\end{aligned}
$$

coincide along $\mathcal{D}_{i j}$. This finishes the proof of the proposition.

We end our paper with a result which will be used in the computation of the mixed Hodge structures associated with multiple logarithms. 
Proposition 5.5. Let $n>1$. For any $1 \leq a<b \leq n$ set $F_{a a}=1$ and

$$
F_{a b}(\mathbf{x})=\mathfrak{L}_{b-a}\left(\frac{1-x_{a} x_{a+1}}{1-x_{a}}, \cdots, \frac{1-x_{a} \cdots x_{b}}{1-x_{a} \cdots x_{b-1}}\right) .
$$

Let $1 \leq j<n$ and $q_{j 0} \in \pi_{1}\left(S_{n}, \mathbf{x}\right)$ (resp. $1 \leq j<n$ and $q_{1 j}, 2 \leq j \leq n$ and $q_{j n}$ ) be a loop turning around the component $\mathcal{D}_{j 0}=\left\{x_{j}=0\right\}$ (resp. $\mathcal{D}_{1 j}=\left\{x_{1} \cdots x_{j}=1\right\}$, resp. $\left.\mathcal{D}_{j n}=\left\{x_{j} \cdots x_{n}=1\right\}\right)$, only once but no other irreducible components of $X_{n}$ such that $\int_{q_{j 0}} d x_{j} / x_{j}=2 \pi i$ (resp. $\int_{q_{1 j}} d \log \left(1-x_{1} \cdots x_{j}\right)=2 \pi i$, resp. $\left.\int_{q_{j n}} d \log \left(1-x_{j} \cdots x_{n}\right)=2 \pi i\right)$. Then

$$
\begin{aligned}
& \left(\Theta\left(q_{j 0}\right)-\mathrm{id}\right) F_{1 n}(\mathbf{x})=-2 \pi i \sum_{s=j}^{n-1} F_{1 s}(\mathbf{x}) F_{s+1, n}(\mathbf{x}), \\
& \left(\Theta\left(q_{1 j}\right)-\mathrm{id}\right) F_{1 n}(\mathbf{x})=2 \pi i F_{1, j}(\mathbf{x}) F_{j+1, n}(\mathbf{x}) \\
& \left(\Theta\left(q_{j n}\right)-\mathrm{id}\right) F_{1 n}(\mathbf{x})=-2 \pi i F_{1, j-1}(\mathbf{x}) F_{j n}(\mathbf{x}),
\end{aligned}
$$

where $\Theta(q)$ denotes the action of $q \in \pi\left(S_{n}, \mathbf{x}\right)$.

Proof. The proposition follows from the monodromy property of $\mathfrak{L}_{n}(\mathbf{x})$. We only prove the result for $\mathcal{D}_{j 0}$ because the proof is exactly the same for $\mathcal{D}_{1 j}$ and $\mathcal{D}_{j n}$.

By Theorem 5.3 we know that the monodromy of $\mathfrak{L}_{n}(\mathbf{x})$ around $x_{s} \cdots x_{n}=1$ is given by $-2 \pi i \mathfrak{L}_{s-1}\left(x_{1}, \ldots, x_{s-1}\right) F_{s n}(\mathbf{x})$. Let $y_{s}=\frac{1-x_{1} \cdots x_{s+1}}{1-x_{1} \cdots x_{s}}$ and $\mathbf{y}=\left(y_{1}, \ldots, y_{n-1}\right)$. On $\mathcal{D}_{j 0}$ all of $y_{s} \cdots y_{n-1}=\frac{1-x_{1} \cdots x_{n}}{1-x_{1} \cdots x_{s}}, j \leq s<n$, are equal to 1 . Therefore the monodromy of $F_{1 n}(\mathbf{x})=\mathfrak{L}_{n-1}(\mathbf{y})$ about $\mathcal{D}_{j 0}$ is the sum

$$
-2 \pi i \sum_{s=j}^{n-1} \mathfrak{L}_{s-1}\left(y_{1}, \ldots, y_{s-1}\right) F_{s, n-1}(\mathbf{y})=-2 \pi i \sum_{s=j}^{n-1} F_{1 s}(\mathbf{x}) F_{s+1, n}(\mathbf{x}) .
$$

This concludes our proposition and the paper.

\section{References}

[1] A. A. Beilinson and P. Deligne, Interprétation motivique de la conjecture de Zagier reliant polylogarithmes et régulateurs, in: Proc. Sym. Pure Math. 55 part 2, Amer. Math. Soc. (1994), 97-121.

[2] A. A. Beilinson, A. B. Goncharov, V. V. Schechtman, and A. N. Varchenko, Aomoto dilogarithms, mixed Hodge structures and motivic cohomology of pairs of triangles in the plane, in: Grothendieck Festschrift II, Prog. in Math. 87, Birkhäuser, Boston, 1991, $78-131$.

[3] K.-T.-Chen, Algebras of iterated path integrals and fundamental groups, Trans. Amer. Math. Soc. 156(1971), 359-379.

[4] K.-T.-Chen, Iterated path integrals, Bull. AMS 83(1977), 831-879.

[5] P. Deligne, Letter to Spencer Bloch, April 3, 1984.

[6] A. B. Goncharov, Polylogarithms in arithmetic and geometry, in: Proc. ICM, Zürich, 374-387, Vol. I, Birkhäuser, 1994.

[7] A. B. Goncharov, The double logarithm and Manin's complex for modular curves, Math. Res. Letters 4(1997), 617-636.

[8] A. B. Goncharov, Multiple $\zeta$-values, Galois groups, and geometry of modular varieties, available online http://xxx.lanl.gov/abs/math.AG/0005069 
[9] R. Hain, Classical polylogarithms, in: Proc. Sym. Pure Math. 55 part 2, Amer. Math. Soc. (1994), 3-42.

[10] R. Hain and R. MacPherson, Higher logarithms, Ill. J. Math. 34(1990), 392-475.

[11] H. Poincaré, Oevres, vol. 2, Paris, 1916.

[12] D. Ramakrishnan, On the monodromy of higher logarithms, Proc. AMS 85(1982), 596599 .

[13] R. Ree, Lie elements and an algebra associated with shuffles, Ann. of Math., 68(1958), 210-220.

[14] Z. Wojtkowiak, Mixed Hodge structures and iterated integrals I, preprint.

[15] D. Zagier, The Block-Wigner-Ramakrishnan polylogarithm function, Math. Ann. 286(1990), 613-624.

[16] J. Zhao, Multiple polylogarithms: analytic continuation, monodromy, and variations of mixed Hodge structures, in "Contemporary Trends in Algebraic Geometry and Algebraic Topology," edited by S.S. Chern, L. Fu and R. Hain, Nankai Tracts in Mathematics, vol. 5, pp. 167-193, World Scientific, 2002.

Address: Department of Mathematics, University of Pennsylvania, PA 19104, USA

Email: jqz@math.upenn.edu 